\title{
Infiltrating Ductal Carcinoma T4b Classification - Medical Images
}

\section{David Gicheha Njoroge ${ }^{1,2 *}$}

${ }^{1}$ Pathology Department, The Karen Hospital, Nairobi, Kenya

${ }^{2}$ Cyto Hist Systems, East Africa, Nairobi, Kenya

Nonmetastatic non-inflammatory invasive breast cancers having skin involvement (SI) area unit classified as T4b, no matter size. This study evaluated malady specific survival (DSS) to work out whether or not size ought to be thought of for these lesions, instead of grouping all into Stage III $[1,2]$.

Here we observed the Infiltrating ductal carcinoma T4b microscopic and Gross section. With microscopic section we observed infiltrating extremely organic phenomenon hyperchromatic cells with sac nuclei and distinguished nucleoliand lympho tube invasion. Nottingham combined microscopic anatomy score three with growth space forming cannular structures (40x; field diameter $0.50 \mathrm{~mm}$. overall grade scores

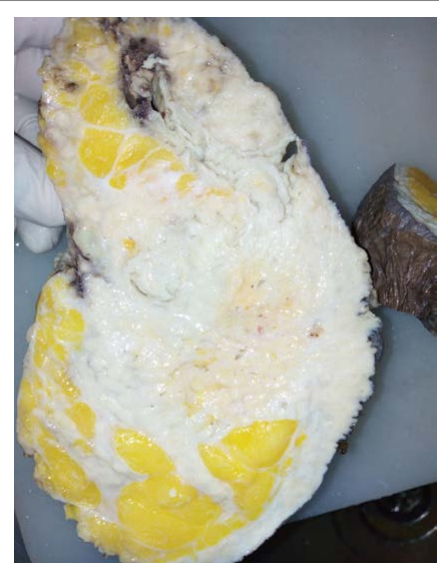

Figure 1: Microscopy - shows infiltrating highly pleomorphic hyperchromatic cells with vesicular nuclei and prominent nucleoli and lympho vascular invasion. Nottingham combined histological score 3 with tumour area forming tubular structures (40x; field diameter $0.50 \mathrm{~mm}$. overall grade scores 8 or 9 ). The invasive carcinoma directly invades the dermis with skin ulceration classified as T4B.

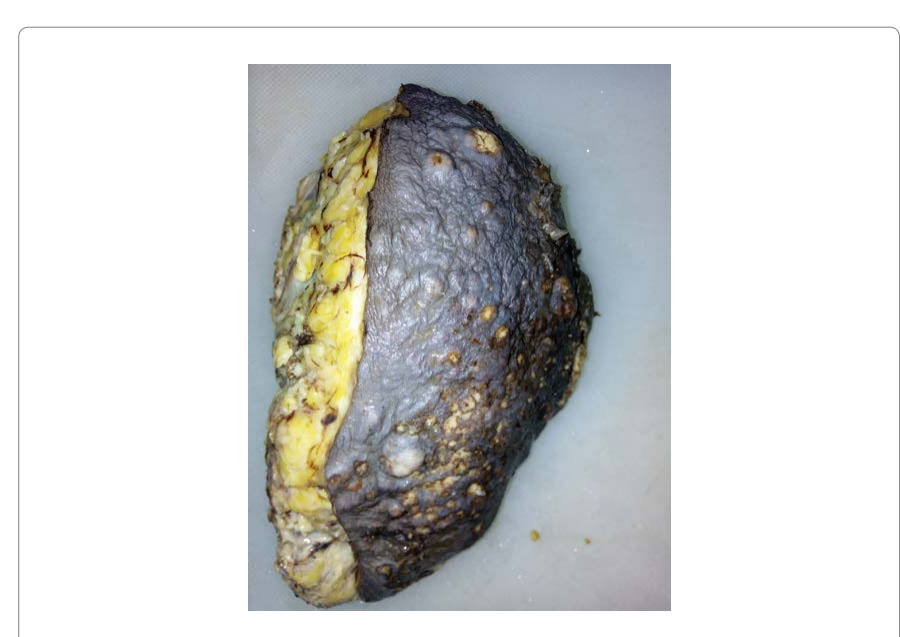

Figure 2: Gross - Total mastectomy specimen measuring $22 \times 12 \times 8 \mathrm{~cm}$. The skin show ulcer measuring $3 \mathrm{~cm}$ together with multiple whitish gray nodules affecting all quadrants. The cut surface shows tumour area measuring $10 \mathrm{~cm}$ diameter.

eight or nine. The invasive malignant neoplastic disease directly invades the corium with skin ulceration classified as T4B and also with Gross section. The skin show ulcer measuring $3 \mathrm{~cm}$ together with multiple whitish gray nodules affecting all quadrants (Figures 1 and 2).

\section{References}

1. Leste SC, Bose S, Chen YY, Connolly JL, de Bacca ME, et al. (2016) Baca Protocol for the Examination of Specimens From Patients With Invasive Carcinoma of the Breast, AJCC/UICC TNM (7thedn), College of American Pathologists, USA.

2. Singletary SE, Connolly JL (2006) Breast cancer staging: working with the sixth edition of the AJCC Cancer Staging Manual. CA Cancer J Clin 56: 37-47.
*Corresponding author: David Gicheha Njoroge, Pathology Department, The Karen Hospital, Nairobi, Kenya, Tel: +254206613000; E-mail: dngicheha.dgn1fteh@gmail.com

Received November 23, 2017; Accepted January 08, 2018; Published January 17,2018

Citation: Njoroge DG (2018) Infiltrating Ductal Carcinoma T4b Classification Medical Images. J Cytol Histol 9: 498. doi: 10.4172/2157-7099.1000498

Copyright: () 2018 Njoroge DG. This is an open-access article distributed under the terms of the Creative Commons Attribution License, which permits unrestricted use, distribution, and reproduction in any medium, provided the original author and source are credited. 